\begin{tabular}{|c|c|}
\hline Title & Complex A djoint V ariable Method for Finite Element A nalysis of Eddy Current Problems \\
\hline Author(s) & Igarashi, Hajime; Watanabe, Kota \\
\hline Citation & $\begin{array}{l}\text { IEEE Transactions on Magnetics, 46(8), 2739-2742 } \\
\text { https://doi.org/10.1109/T MA G.2010.2043936 }\end{array}$ \\
\hline Issue Date & $2010-08$ \\
\hline Doc URL & http:/hdl.handle.net/2115/45008 \\
\hline Rights & $\begin{array}{l}\text { () 2010 IEEE. Personal use of this material is permitted. However, permission to reprint/republish this material for } \\
\text { advertising or promotional purposes or for creating new collective works for resale or redistribution to servers or lists, } \\
\text { or to reuse any copyrighted component of this work in other works must be obtained from the IEEE. }\end{array}$ \\
\hline Type & article \\
\hline File Information & IEEETM46-8_2739-2742.pdf \\
\hline
\end{tabular}

Instructions for use 


\title{
Complex Adjoint Variable Method for Finite-Element Analysis of Eddy Current Problems
}

\author{
Hajime Igarashi and Kota Watanabe \\ Graduate School of Information Science and Technology, Hokkaido University, Sapporo 060-0814, Japan
}

\begin{abstract}
This paper presents the adjoint variable method (AVM) for finite-element (FE) analysis of eddy current problems based on complex variables. In the sensitivity analysis based on FE analysis of time-harmonic eddy current fields, the functions for which sensitivity is evaluated are often real-valued, while unknown variables in the FE analysis are complex. When the AVM is applied to such problems, the real-valued functions are differentiated with respect to the complex variables. However, such differentiation cannot be defined because the Cauchy-Riemann equation does not hold. In this paper, the AVM for complex systems is introduced and applied to linear and nonlinear eddy current problems, in the latter of which the harmonic balance method is employed.
\end{abstract}

Index Terms-Adjoint variable method, finite-element method (FEM), fixed point method, harmonic balance, sensitivity analysis.

\section{INTRODUCTION}

$\mathbf{T}$ HE adjoint variable method (AVM) has widely been applied to various problems in computational electromagnetism to compute sensitivity of global and field quantities in the post processing as well as to evaluate derivative of objective functions in optimization and inverse problems [1]-[3]. It is one of the advantages in AVM that its computational costs are almost independent of the number of design parameters by which objective quantities are differentiated in contrast to finite difference (FD) computations of the derivative.

In AVM, the adjoint equation must be solved before the computation of sensitivity. The right-hand side vector in the adjoint equation is composed of the derivatives of the objective quantity with respect to the unknown variable of the field analysis. When time-harmonic eddy current fields are analyzed using finite-element method (FEM), the unknown variables are complex. On the other hand, the objective quantities are real-valued when they express, for example, energy, loss and effective values of field variables. In such cases, the derivative cannot be defined because the Cauchy-Riemann equation does not hold. The similar situations can also be found in microwave problems [4] as well as other field problems.

In this paper, the AVM for complex systems will be introduced by adequate modification of the conventional AVM and be applied to sensitivity analysis of linear and nonlinear eddy current problems, in the latter of which the harmonic balance technique with the fixed point method [5] is employed.

\section{Linear EdDy CURRENT PRoblems}

To address the problem clearly, let us consider first a steadystate linear eddy current problem formulated with A method in which

$$
\operatorname{rot}(\nu \operatorname{rot} A)+\mathrm{j} \omega \sigma A=\boldsymbol{J}
$$

Manuscript received December 23, 2009; accepted February 10, 2010. Current version published July 21, 2010. Corresponding author: H. Igarashi (e-mail: igarashi@ssi.ist.hokudai.ac.jp).

Color versions of one or more of the figures in this paper are available online at http://ieeexplore.ieee.org.

Digital Object Identifier 10.1109/TMAG.2010.2043936 is solved, where $\boldsymbol{A}$ and $\boldsymbol{J}$ are vector potential and current density, $\nu, \omega$ and $\sigma$ are the inverse of magnetic permeability which is constant, angular frequency and conductivity. The edge element-based FE method applied to (1) yields a system of linear equations

$$
\mathrm{K} \boldsymbol{a}=\boldsymbol{b}
$$

where $\mathrm{K} \in \mathbb{C}^{E \times E}, \boldsymbol{a} \in \mathbb{C}^{E}, \boldsymbol{b} \in \mathbb{R}^{E}$ and $E$ denotes the number of edges in the FE mesh. The FE matrix is decomposed as $\mathrm{K}=$ $\mathrm{N}+\mathrm{j} \omega \mathrm{S}$ whose entities as well as those of $\boldsymbol{b}$ are given by

$$
\begin{aligned}
\mathrm{N}_{i j} & =\int_{v} \nu \operatorname{rot} \boldsymbol{N}_{i} \cdot \operatorname{rot} \boldsymbol{N}_{j} d v \\
\mathrm{~S}_{i j} & =\int_{v} \sigma \boldsymbol{N}_{i} \cdot \boldsymbol{N}_{j} d v \\
b_{i} & =\int_{v} \boldsymbol{N}_{i} \cdot \boldsymbol{J} d v
\end{aligned}
$$

where $N_{i}$ is the basis function for the edge-based element.

Now, it is assumed that the electromagnetic field depends on finite number of parameters $\boldsymbol{p}=\left[p_{1}, p_{2}, \ldots, p_{n}\right]^{t}$, which express, for example, device shapes and material characteristics. In this situation, we can write $\boldsymbol{a}=\boldsymbol{a}(\boldsymbol{p})$. Moreover, let us consider the numerical evaluation of sensitivity $\partial W / \partial p_{k}$, $k=1,2, \ldots, n$ for a real valued function $W(\boldsymbol{a})$. In practical applications, the function $W$ expresses quantities such as electromagnetic energy, loss, and effective values of magnetic induction. In order to evaluate the sensitivity effectively, we employ the AVM, where the adjoint equation to (2)

$$
\mathrm{K} \overline{\boldsymbol{a}}=\frac{\partial W}{\partial \boldsymbol{a}}
$$

is solved to obtain the adjoint variable $\overline{\boldsymbol{a}}$. As shown below, by solving (2), (4), the sensitivity can be computed from $\boldsymbol{a}$ and $\overline{\boldsymbol{a}}$. However, the right-hand side of (4), which is the derivative of the real-valued function with respect to the complex variable, cannot be defined because the function $W$ does not satisfy the Cauchy-Riemann differential equation

$$
\frac{\partial W_{r}}{\partial \boldsymbol{x}}=\frac{\partial W_{i}}{\partial \boldsymbol{y}}, \quad \frac{\partial W_{r}}{\partial \boldsymbol{y}}=-\frac{\partial W_{i}}{\partial \boldsymbol{x}}
$$

where the indices $r$ and $i$ denote real and imaginary components, and $\boldsymbol{x}=\operatorname{Re}(\boldsymbol{a}), \boldsymbol{y}=\operatorname{Im}(\boldsymbol{a})$. Similar notations will be used 
hereafter. For example, when we compute the sensitivity in the magnetic energy, that is

$$
W=\frac{1}{2} \sum_{i}^{E} \sum_{j}^{E} a_{i} a_{j}^{*} \int_{v} \nu \operatorname{rot} \boldsymbol{N}_{i} \cdot \operatorname{rot} \boldsymbol{N}_{j} d v
$$

it is obvious that (5) does not hold. To overcome this difficulty, we will modify the adjoint equation below. Assuming that $W$ is differentiable by the real variables, $x$ and $y$, its sensitivity with respect to a parameter $p$ can be computed from

$$
\frac{\partial W}{\partial p}=\left(\frac{\partial \boldsymbol{x}}{\partial p}\right)^{t} \frac{\partial W}{\partial \boldsymbol{x}}+\left(\frac{\partial \boldsymbol{y}}{\partial p}\right)^{t} \frac{\partial W}{\partial \boldsymbol{y}} .
$$

Note that (7) becomes

$$
\frac{\partial W}{\partial p}=\left(\frac{\partial \boldsymbol{a}}{\partial p}\right)^{t} \frac{\partial W}{\partial \boldsymbol{a}}
$$

if (5) holds. It would be computationally ineffective to perform FD approximation for, e.g., $\partial \boldsymbol{x} / \partial p$ when the sensitivity (7) must be evaluated for more than a few parameters. To evaluate the sensitivity more effectively, the AVM is employed. By taking differentiation of (2) with respect to $p$ under the assumption that $\boldsymbol{b}$ is independent of $p$, we have

$$
\mathrm{K} \frac{\partial \boldsymbol{a}}{\partial p}=-\frac{\partial \mathrm{K}}{\partial p} \boldsymbol{a} .
$$

Taking the inner product of both sides of (9) with an adjoint variable $\overline{\boldsymbol{a}}$ and taking its transpose, we have

$$
\left(\frac{\partial \boldsymbol{a}}{\partial p}\right)^{t} \mathrm{~K} \overline{\boldsymbol{a}}=-\boldsymbol{a}^{t} \frac{\partial \mathrm{K}}{\partial p} \overline{\boldsymbol{a}} .
$$

Now, it can be found that if

$$
\mathrm{K} \overline{\boldsymbol{a}}=\frac{\partial W}{\partial \boldsymbol{x}}-\mathrm{j} \frac{\partial W}{\partial \boldsymbol{y}}
$$

holds, the real part of the right-hand side of (10) coincides with (7). Thus, the sensitivity can be computed from

$$
\frac{\partial W}{\partial p}=-\operatorname{Re}\left(\boldsymbol{a}^{t} \frac{\partial \mathrm{K}}{\partial p} \overline{\boldsymbol{a}}\right) .
$$

A formula similar to (12) has been obtained for microwave circuit problems [6].

\section{Nonlinear EdDy CURRENT PROBlems}

In this section, the complex AVM presented in the previous section is extended to nonlinear eddy current problems.

\section{A. Harmonic Balance With Fixed Point Method}

To consider the nonlinear eddy current problems in which magnetic saturation is taken into account, the harmonic balance method based on the fixed point method [5], governed by

$$
\nu_{F} \operatorname{rotrot} \boldsymbol{A}+\sigma \frac{\partial \boldsymbol{A}}{\partial t}=\boldsymbol{J}+\operatorname{rot} \boldsymbol{M}_{F}(\boldsymbol{B})
$$

is employed in this work, where $\nu_{F}$ is a constant which has a significant influence on convergence of this method, and $\boldsymbol{M}_{F}$ is the magnetization-like vector which satisfies

$$
\boldsymbol{H}(\boldsymbol{B})=\nu_{F} \boldsymbol{B}-\boldsymbol{M}_{F}(\boldsymbol{B}) .
$$

Note that $\boldsymbol{M}_{F}$ identifies with the magnetization vector when $\nu_{F}=\nu_{0}$. One of the merits of this method is that the equations for each Fourier mode are decoupled so that the size of system equations is effectively reduced in comparison with the original harmonic balance method which solves the coupled equation for all the Fourier modes.

Finite-element discretization of (13) with the Fourier decomposition of the unknowns

$$
\boldsymbol{a}(t)=\frac{\boldsymbol{a}_{0}}{2}+\operatorname{Re}\left(\sum_{n=1}^{N} \boldsymbol{a}_{n} e^{\mathrm{j} n \omega t}\right)
$$

results in the semi-decoupled equation

$$
\mathrm{K}_{n} \boldsymbol{a}_{n}=\boldsymbol{g}_{n}\left(\boldsymbol{a}_{0}, \boldsymbol{a}_{1}, \ldots, \boldsymbol{a}_{N}\right)
$$

for $n=0,1,2, \ldots, N$ where $\mathrm{K}_{n}=\mathrm{N}_{F}+\mathrm{j} n \omega \mathrm{S}$. In (16), $\nu$ is replaced by $\nu_{F}$ in the first equation of (3) to obtain $\mathrm{N}_{F}$, and $\boldsymbol{g}_{n}$ is defined by

$$
\begin{aligned}
\boldsymbol{g}_{n} & =\frac{2}{T} \int_{0}^{T} \boldsymbol{g}(t) e^{-\mathrm{j} n \omega t} d t \\
& \simeq \frac{2}{M} \sum_{k=0}^{M-1} \boldsymbol{g}(k T / M) e^{-\mathrm{j} 2 \pi k n / M}
\end{aligned}
$$

where the entities of $\boldsymbol{g}(t)$ are given by

$$
g_{i}(t)=\int_{v}\left(\boldsymbol{N}_{i} \cdot \boldsymbol{J}+\boldsymbol{M}_{F}(\boldsymbol{B}) \cdot \operatorname{rot} \boldsymbol{N}_{i}\right) d v
$$

$i=1,2, \ldots, E, T$ and $M$ denote the time period of the fundamental wave and number of divisions per $T$. In the computation, (16) are solved to obtain $\boldsymbol{a}_{n}$ for $n=0,1,2, \ldots, N$ which are inserted to (15) to have $\boldsymbol{a}(t)$. Then, $\boldsymbol{B}(t)$ as well as $\boldsymbol{M}_{F}(t)$ are computed from (14), where $\boldsymbol{H}(\boldsymbol{B})$ is obtained from the assumed BH curve. Moreover, $\boldsymbol{g}_{n}$ is computed from (17) and (18) to be substituted into the right-hand side of (16). These iterative processes are continued until convergence.

\section{B. Complex Adjoint Equation for Nonlinear Systems}

We will formulate the complex AVM for the nonlinear eddy current problem (16). In this case, the sensitivity for a function $W=W\left(\boldsymbol{a}_{0}, \boldsymbol{a}_{1}, \ldots, \boldsymbol{a}_{N}\right)$ can be obtained by

$$
\frac{\partial W}{\partial p}=\sum_{n=0}^{N}\left[\left(\frac{\partial \boldsymbol{x}_{n}}{\partial p}\right)^{t} \frac{\partial W}{\partial \boldsymbol{x}_{n}}+\left(\frac{\partial \boldsymbol{y}_{n}}{\partial p}\right)^{t} \frac{\partial W}{\partial \boldsymbol{y}_{n}}\right] .
$$

The AVM will be formulated to make effective evaluation of (19). Differentiation of (16) with respect to $p$ yields

$$
\mathrm{K}_{n} \frac{\partial \boldsymbol{a}_{n}}{\partial p}-\sum_{m=0}^{N}\left(\mathrm{X}_{n m} \frac{\partial \boldsymbol{x}_{m}}{\partial p}+\mathrm{Y}_{n m} \frac{\partial \boldsymbol{y}_{m}}{\partial p}\right)=-\frac{\partial \mathrm{K}_{n}}{\partial p} \boldsymbol{a}_{n}
$$


for $n=0,1,2, \ldots, N$, where $\mathrm{X}_{n m}=\partial \boldsymbol{g}_{n} / \partial \boldsymbol{x}_{m}, \mathrm{Y}_{n m}=$ $\partial \boldsymbol{g}_{n} / \partial \boldsymbol{y}_{m}$, which will be shown to be symmetric but not commutable between $n$ and $m$. In derivation of (20), it is assumed that $\boldsymbol{J}$ and $M_{F}$ are not explicit functions of $p$. In a matrix form, (20) can be expressed as

$$
(\mathrm{K}-\mathrm{X}) \frac{\partial \boldsymbol{x}}{\partial p}+(\mathrm{jK}-\mathrm{Y}) \frac{\partial \boldsymbol{y}}{\partial p}=-\frac{\partial \mathrm{K}}{\partial p} \boldsymbol{a}
$$

where $\boldsymbol{a}=\left[\boldsymbol{a}_{0}, \boldsymbol{a}_{1}, \ldots, \boldsymbol{a}_{N}\right]^{t}$, and $\mathrm{K}, \mathrm{X}$, and $\mathrm{Y}$ are the matrices expressing relations among the Fourier components, which are defined by $\mathrm{K}=\operatorname{diag}\left(\mathrm{K}_{0}, \mathrm{~K}_{1}, \ldots, \mathrm{K}_{N}\right)$

$$
\mathrm{X}=\left[\begin{array}{cccc}
\mathrm{X}_{00} & X_{01} & \cdots & \mathrm{X}_{0 N} \\
\mathrm{X}_{10} & \ddots & & \\
\vdots & & & \\
\mathrm{X}_{N 0} & \cdots & & \mathrm{X}_{N N}
\end{array}\right]
$$

and $\mathrm{Y}$ is similarly defined.

Introducing an adjoint variable $\overline{\boldsymbol{a}} \in \mathbb{C}^{E N}$, and taking an inner product between $\overline{\boldsymbol{a}}$ and (21), we have

$$
\left(\frac{\partial \boldsymbol{x}}{\partial p}\right)^{t}\left(\mathrm{~K}-\mathrm{X}^{t}\right) \overline{\boldsymbol{a}}+\left(\frac{\partial \boldsymbol{y}}{\partial p}\right)^{t}\left(\mathrm{jK}-\mathrm{Y}^{t}\right) \overline{\boldsymbol{a}}=-\boldsymbol{a}^{t} \frac{\partial \mathrm{K}}{\partial p} \overline{\boldsymbol{a}} \text {. }
$$

Hence, it can be found from (19) and (23) that the sensitivity can be obtained from

$$
\frac{\partial W}{\partial p}=-\operatorname{Re}\left(\sum_{n=0}^{N} \boldsymbol{a}_{n}^{t} \frac{\partial \mathrm{K}_{n}}{\partial p} \overline{\boldsymbol{a}}_{n}\right)
$$

if $\overline{\boldsymbol{a}}$ satisfies

$$
\begin{aligned}
\operatorname{Re}\left\{\left(\mathrm{K}-\mathrm{X}^{t}\right) \overline{\boldsymbol{a}}\right\} & =\frac{\partial W}{\partial \boldsymbol{x}} \\
\operatorname{Re}\left\{\left(\mathrm{jK}-\mathrm{Y}^{t}\right) \overline{\boldsymbol{a}}\right\} & =\frac{\partial W}{\partial \boldsymbol{y}}
\end{aligned}
$$

which can also be expressed as

$$
\left[\begin{array}{cc}
\mathrm{K}_{r}-\mathrm{X}_{r}^{t} & -\mathrm{K}_{i}^{t}+\mathrm{X}_{i}^{t} \\
\mathrm{~K}_{i}+\mathrm{Y}_{r}^{t} & \mathrm{~K}_{r}^{t}-\mathrm{Y}_{i}^{t}
\end{array}\right]\left[\begin{array}{l}
\overline{\boldsymbol{x}} \\
\overline{\boldsymbol{y}}
\end{array}\right]=\left[\begin{array}{c}
\partial W / \partial \boldsymbol{x} \\
-\partial W / \partial \boldsymbol{y}
\end{array}\right] .
$$

The adjoint variable $\overline{\boldsymbol{a}}$, which contains all the Fourier components, can be obtained by solving (26).

The solution of the coupled (26) would become impractical when the number of involved Fourier components increases. To overcome this difficulty, the coupling matrices $\mathrm{X}, \mathrm{Y}$ in (26) are moved to the right-hand side to obtain

$$
\mathrm{K}_{n} \overline{\boldsymbol{a}}_{n}=\frac{\partial W}{\partial \boldsymbol{x}_{n}}-\mathrm{j} \frac{\partial W}{\partial \boldsymbol{y}_{n}}+\sum_{m=0}^{N}\left\{\operatorname{Re}\left(\mathrm{X}_{m n} \overline{\boldsymbol{a}}_{m}\right)-\mathrm{j} \operatorname{Re}\left(\mathrm{Y}_{m n} \overline{\boldsymbol{a}}_{m}\right)\right\}
$$

for $n=0,1, \ldots, N$, which can be seen as the generalization of (11). Equation (27) could be solved by the Jacobi-type iterations; at the first step, each equation in which the coupling terms are neglected is solved for $\overline{\boldsymbol{x}}_{n}^{1}, \overline{\boldsymbol{y}}_{n}^{1}$, then the coupling terms are evaluated using $\overline{\boldsymbol{x}}_{n}^{1}, \overline{\boldsymbol{y}}_{n}^{1}$ in the equations which are solved for $\overline{\boldsymbol{x}}_{n}^{2}$, $\overline{\boldsymbol{y}}_{n}^{2}$.

\section{Evaluation of Coupling Matrices}

This section derives the explicit forms for $\mathrm{X}_{n m}, \mathrm{Y}_{n m}$, the former of which is given by

$$
\mathrm{X}_{n m}=\frac{2}{T} \int_{0}^{T} \frac{\partial \boldsymbol{g}(t)}{\partial \boldsymbol{x}_{m}} e^{-\mathrm{j} n \omega t}
$$

where the $i$ th entity of $\partial \boldsymbol{g}(t) / \partial \boldsymbol{x}_{m}$ is given by

$$
\frac{\partial g_{i}(t)}{\partial \boldsymbol{x}_{m}}=\int_{v} \frac{\partial \boldsymbol{M}_{F}}{\partial \boldsymbol{x}_{m}} \cdot \operatorname{rot} \boldsymbol{N}_{i} d v .
$$

Assuming that $\boldsymbol{M}_{F}$ is expressed by $\boldsymbol{M}_{F}=\tilde{\nu}_{F}\left(B^{2}\right) \boldsymbol{B}$, $\partial \boldsymbol{M}_{F} / \partial \boldsymbol{x}_{m}$ can be written as

$$
\frac{\partial \boldsymbol{M}_{F}}{\partial \boldsymbol{x}_{m}}=\frac{\partial \tilde{\nu}_{F}}{\partial B^{2}} \frac{\partial B^{2}}{\partial \boldsymbol{x}_{m}} \boldsymbol{B}+\tilde{\nu}_{F} \frac{\partial \boldsymbol{B}}{\partial \boldsymbol{x}_{m}} .
$$

The derivatives $\partial B^{2} / \partial \boldsymbol{x}_{m}, \partial \boldsymbol{B} / \partial \boldsymbol{x}_{m}$ in (30) are shown to be given by

$$
\begin{aligned}
& \frac{\partial B^{2}}{\partial \boldsymbol{x}_{m}}=2 d_{m}\left[\boldsymbol{B} \cdot \operatorname{rot} \boldsymbol{N}_{1}, \boldsymbol{B} \cdot \operatorname{rot} \boldsymbol{N}_{2}, \ldots, \boldsymbol{B} \cdot \operatorname{rot} \boldsymbol{N}_{E}\right]^{t} \\
& \text { and } \\
& \frac{\partial \boldsymbol{B}}{\partial \boldsymbol{x}_{m}}=d_{m}\left[\operatorname{rot} \boldsymbol{N}_{1}, \operatorname{rot} \boldsymbol{N}_{2}, \ldots, \operatorname{rot} \boldsymbol{N}_{E}\right]^{t}
\end{aligned}
$$

where $d_{m}=1 / 2$ if $m=0$, otherwise $d_{m}=\cos (m \omega t)$. Consequently, the entities of the matrix $\partial \boldsymbol{g}(t) / \partial \boldsymbol{x}_{m}$ are given by

$$
\begin{array}{r}
\left(\frac{\partial \boldsymbol{g}}{\partial \boldsymbol{x}_{m}}\right)_{i j}=2 d_{m} \int_{v} \frac{\partial \tilde{\nu}_{F}}{\partial B^{2}}\left(\boldsymbol{B} \cdot \operatorname{rot} \boldsymbol{N}_{i}\right)\left(\boldsymbol{B} \cdot \operatorname{rot} \boldsymbol{N}_{j}\right) d v \\
+d_{m} \int_{v} \tilde{\nu}_{F}\left(\operatorname{rot} \boldsymbol{N}_{i} \cdot \operatorname{rot} \boldsymbol{N}_{j}\right) d v .
\end{array}
$$

It can be shown that replacing $d_{m}$ in (33) by $-\sin (m \omega t)$, we can have the corresponding formula for $\partial \boldsymbol{g}(t) / \partial \boldsymbol{y}_{m}$.

When there is no magnetic saturation, that is, $\tilde{\nu}_{F}$ is constant, it can be found from (28) and (33) that $\mathrm{X}_{m n}$ and $\mathrm{Y}_{m n}$ vanish for $m \neq n$, and otherwise

$$
\mathrm{X}_{m n}=\int_{v} \tilde{\nu}_{F}\left(\operatorname{rot} \boldsymbol{N}_{i} \cdot \operatorname{rot} \boldsymbol{N}_{j}\right) d v, \quad \mathrm{Y}_{n m}=\mathrm{jX}_{n m} .
$$

Insertion of (34) into (27) yields the decoupled adjoint equation of the form

$$
\mathrm{K}_{n} \overline{\boldsymbol{a}}_{n}=\frac{\partial W}{\partial \boldsymbol{x}_{n}}-\mathrm{j} \frac{\partial W}{\partial \boldsymbol{y}_{n}}
$$

which is the same as (11) as expected, where $K_{n}$ is redefined by $\mathrm{K}_{n}=\mathrm{N}+\mathrm{j} n \omega \mathrm{S}$.

\section{NUMERICAL EXAMPLES}

\section{A. Toy Problem}

For an illustrative example, a simple problem

$$
\left[\begin{array}{ll}
1 & 2 \\
2 & 1
\end{array}\right]\left[\begin{array}{l}
a_{1} \\
a_{2}
\end{array}\right]+\left[\begin{array}{l}
\sigma \dot{a}_{1} \\
\sigma \dot{a}_{2}
\end{array}\right]=-\left[\begin{array}{l}
c_{1} a_{1}^{3} \\
c_{2} a_{2}^{3}
\end{array}\right]+\left[\begin{array}{l}
\omega \cos \omega t \\
\omega \cos \omega t
\end{array}\right]
$$

is considered. When $c_{1}=c_{2}=0$, (36) becomes linear equations for which monochromatic equations like (1) can be derived and analytical solutions can be obtained. The energy function 


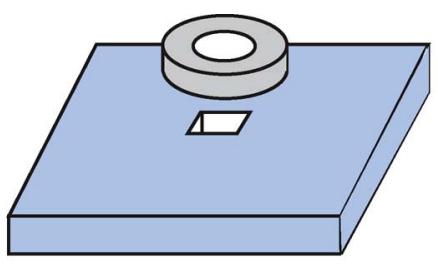

(a)

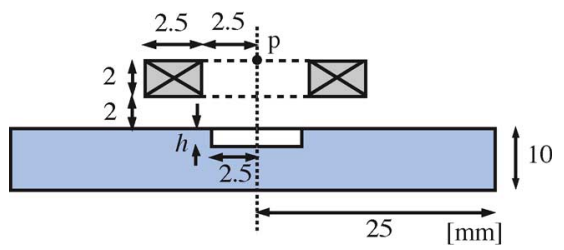

(b)

Fig. 1. Coil and aluminum plate containing a flaw. (a) Schematic view. (b) Cross-sectional view.

TABLE I

COMPARISON OF RESULTANT SENSITIVITY

\begin{tabular}{ccccc}
\hline method & present & \multicolumn{3}{c}{ finite difference } \\
\hline$\Delta h[\mathrm{~mm}]$ & - & 0.02 & 0.01 & 0.005 \\
$d B / d h$ & 0.1297 & 0.1209 & 0.1214 & 0.1279 \\
\hline
\end{tabular}

$W=\boldsymbol{a a}^{*} / 2$ can be expressed in terms of the complex solution $\boldsymbol{a}$. The sensitivity $d W / d \sigma$ is computed by direct differentiation of $W$ with respect to $\sigma$ to have the result $d W / d \sigma=$ $-2 \omega^{2} \sigma /\left(9+\sigma^{2}\right)^{2}$. On the other hand, by solving the adjoint (11), we have $\overline{\boldsymbol{a}}=\left[\omega /\left(9+\sigma^{2}\right), \omega /\left(9+\sigma^{2}\right)\right]^{t}$, which is substituted to (12) to evaluate $d W / d \sigma$. It is finally found that both results for $d W / d \sigma$ are identical.

The nonlinear equation (36) is then solved using the harmonic balance method mentioned in Section II. The sensitivity in $W=$ $\sum_{n} \boldsymbol{a}_{n}^{*} \boldsymbol{a}_{n}$ is evaluated by the FD method, where (36) is solved twice for different $\sigma$ to compute $\Delta W / \Delta \sigma$, and by the present method. In the computations, we set $\omega=2 \pi, \sigma=1$ and $M=$ 160. As a result, both results for $d W / d \sigma$ are found to agree well being independent of $c_{1}, c_{2}$, for example, both are -0.479 when $c_{1}=8, c_{2}=4$. It is found that the convergence in (16) becomes slow as $c_{1}, c_{2}$ increases, that is, nonlinearity becomes strong.

\section{B. Nondestructive Testing}

The present method is applied to the sensitivity analysis of a nondestructive testing system, shown in Fig. 1. In this model, the alternative current of 1 [kAT] flows along the coil at $10[\mathrm{kHz}]$. The metallic plate contains a rectangular flaw whose thickness $h$ is set to $1[\mathrm{~mm}]$. The sensitivity $d B / d h$ in the magnetic induction $B$ measured at point $\mathrm{p}$ against the thickness $h$ of the flaw is computed using the present method and finite differentiation $\Delta B / \Delta h$. The whole system including the air region is subdivided into 102580 tetrahedral elements with 123555 unknowns.

For a linear problem, the metallic plate is assumed to be made of aluminum, whose conductivity is set to $36 \times 10^{6}[\mathrm{~S} / \mathrm{m}]$. In the present method, the value of $d B / d h$ is computed from (12) after solving the adjoint (11).

The numerical results are summarized in Table I. It is clear that $\Delta B / \Delta h$ approaches $d B / d h$ computed by the present method as $\Delta h$ decreases.

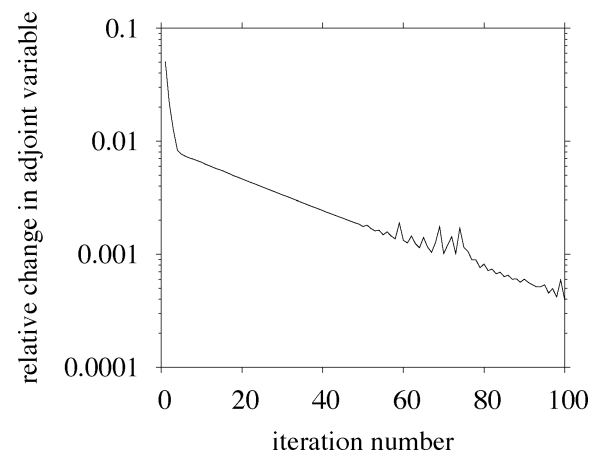

Fig. 2. Convergence of $\overline{\boldsymbol{a}}$ in (27). The vertical axis represents the ratio $\mid \overline{\boldsymbol{a}}^{k+1}-$ $\overline{\boldsymbol{a}}^{k}|/| \overline{\boldsymbol{a}}^{k} \mid$, where $k$ is the iteration number.

For a nonlinear problem, the metallic plate is assumed to be made of the carbon steel S45C whose conductivity is $7 \times 10^{6}$ $[\mathrm{S} / \mathrm{m}]$ and its $\mathrm{BH}$ curve can be found in [7]. The frequency of the coil current is now set to $100[\mathrm{~Hz}]$. In the harmonic balance method, first, third, and fifth harmonics are considered. The convergence for the decoupled adjoint (27) is shown in Fig. 2. The values of $d B_{\mathrm{rms}} / d h$ computed from the FD method, where $\Delta h=0.005[\mathrm{~mm}]$ and the present method are 0.202 and $0.171[\mathrm{~T} / \mathrm{m}]$, respectively. The discrepancy between these results would be due to round-off errors in the FD method, where $\Delta B$ is computed by $(0.17246222-0.17246121) / \Delta h$. In contrast, the result of the present method is not suffered from such round-off errors.

\section{CONCLUSION}

In this paper, the AVM for complex systems has been presented and applied to linear and nonlinear eddy current problems, in the latter of which the harmonic balance technique with the fixed point method is employed. It has been shown that the sensitivity computed by the present method agrees well with those obtained by analytical method and finite difference method. In the last test problem, there is a discrepancy between the results obtained by the present and finite difference method, in the latter of which accuracy would be deteriorated by round-off errors.

\section{REFERENCES}

[1] D.-Y. Jeon, D. Kim, and S.-y. Hahn, "Optimum design of linear synchronous motor using evolution strategy combined with stochastic FEM," IEEE Trans. Magn., vol. 35, no. 3, pp. 1734-1737, May 1999.

[2] H. Akel and J. P. Webb, "Design sensitivities for scattering-matrix calculation with tetrahedral edge elements," IEEE Trans. Magn., vol. 36, no. 4, pp. 1043-1046, Jul. 2000.

[3] Y. Okamoto, K. Akiyama, and N. Takahashi, "3-D topology optimization of single-pole-type head by using design sensitivity analysis," IEEE Trans. Magn., vol. 42, no. 4, pp. 1087-1091, Apr. 2006.

[4] J. Jin, The Finite Element Method in Electromagnetics. New York: Wiley, 2002.

[5] S. Ausserhofer, O. Biro, and K. Preis, "An efficient harmonic balance method for nonlinear eddy-current problems," IEEE Trans. Magn., vol. 43, no. 4, pp. 1229-1232, Apr. 2007.

[6] N. K. Nikolova, J. W. Bandler, and M. H. Bakr, "Adjoint techniques for sensitivity analysis in high-frequency structure CAD," IEEE Trans. Microw. Theory Tech., vol. 52, no. 1, pp. 403-419, Jan. 2004.

[7] B. Yoo, M. Hirano, and K. Hirata, "Fully coupled electro-magneto-mechanical analysis method of magnetostrictive actuator using 3-D finite element method," in Proc. 2008 Int. Conf. Elect. Mach., 2008, Paper ID 1175 . 